\title{
Electromagnetic fields near metal surfaces
}

\author{
R K THAPA, P DAS*, N KAR* and R A LAL ${ }^{\dagger}$ \\ Department of Physics, Pachhunga University College, North Eastern Hill University, Aizawl \\ 796001 , India \\ *Department of Physics, North Bengal University, Darjeeling 734430 India \\ ${ }^{\dagger}$ Department of Chemistry, Tripura University, Agartala 799 004, India \\ MS received 6 March 1992
}

\begin{abstract}
Electromagnetic field variation at the surface region of nearly free electron metal (Al), noble metal ( $\mathrm{Ag}$ ) and transition metals $(\mathrm{Cr}, \mathrm{Rh}, \mathrm{Pd}$ and $\mathrm{Mo}$ ) are studied with respect to the incident photon energy. The electromagnetic field used is the one calculated by Bagchi and Kar using the local frequency dependent dielectric response function. The results so obtained may be of significance in the photoemission scattering cross-section calculations in understanding electronic structure and properties of these metals.
\end{abstract}

Keywords. Photoemission; plasmon energy; electromagnetic fields; dielectric; photocurrent.

\section{Introduction}

Photoemission from solids has long been a source of interest in physics but only recently has its potential as a probe of electronic structure been recognized. It is of fundamental importance since it is connected with the basic interaction of electromagnetic field and solid.

The calculation of electromagnetic fields has been a topic of study and has been given due attention by many scientists earlier. To cite a few examples, Kleiwer (1976) considered the semi-classical infinite barrier model, Forstman and Stenschke (1977) used a hydrodynamical approach while Feibelman (1975a, b) has given the most complete results for jellium. However, all these calculations are applicable only in the case of free electron metal. These fields in the surface region can then form the basis for photoemission calculations.

Here, we have been concerned only with the photon energy dependence of the electromagnetic field. The solids taken for the case study are nearly free electron metal ( $\mathrm{Al})$, noble metal $(\mathrm{Ag})$ and transition metals $(\mathrm{Cr}, \mathrm{Rh}, \mathrm{Pd}$ and $\mathrm{Mo})$. We provide here a very simple way of understanding the spatial variation of the electromagnetic field for the surface region defined by $-a / 2 \leqslant z \leqslant a / 2$ by considering a local frequency dependent dielectric function $\varepsilon(\omega, z)$ described elsewhere (Bagchi and Kar 1978). We shall focus our attention mainly on the calculation of the electromagnetic field variation in the surface region for both below and above the plasmon energy since this is responsible for the enhancement of photoyield compared to the value calculated from the classical Fresnel fields.

\section{Theory}

The model used (Bagchi and Kar 1978) is a fairly simple one. The $z$-direction is assumed to be perpendicular to the nominal surface plane which is chosen to be $z=0$ 
plane. The metal is assumed to occupy all the space to the left of $z=0$ plane. The response of the electromagnetic field is assumed to be bulk-like everywhere except in a surface region which extends over $-a / 2 \leqslant z \leqslant a / 2$. In this region, the model dielectric function is chosen to be a local function which interpolates linearly between the bulk value inside the metal and the vacuum value (unity) outside. The model frequency dependent dielectric function is therefore given by

$$
\varepsilon(\omega, z)=\left\{\begin{array}{lr}
\varepsilon(\omega)=\varepsilon_{1}(\omega)+i \varepsilon_{2}(\omega) & z \leqslant-a / 2 \\
\frac{1}{2}[1+\varepsilon(z)]+[1-\varepsilon(\omega)](z / a), & -a / 2 \leqslant z \leqslant a / 2 \\
1 & z \geqslant a / 2
\end{array}\right.
$$

For complex dielectric function, we have used the experimental values given by Weaver (1987-88). We consider a $p$-polarized light to be incident on the surface plane making angle $\theta_{i}$ with the $z$-axis. For $p$-polarized light, the magnetic field $B(z)=$ $B(\vec{Q}, \omega, z)$ (where $\vec{Q}=\omega / c \sin \theta_{i}$ is small) is in the $y$-direction and it obeys the following equation (Landau et al 1984) with $\varepsilon=\varepsilon(\omega, z)$

$$
\left.\frac{\mathrm{d}}{\mathrm{d} z}\left(\frac{1 \mathrm{~d} B}{\varepsilon}\right) \frac{\omega^{2}}{\mathrm{~d} z}\right)+\left(\frac{Q^{2}}{c^{2}}\right) B=0
$$

The electric field components can be obtained from the magnetic field by using the relation

$$
\begin{aligned}
& E^{x}(\vec{Q}, \omega, z)=\frac{c}{i \omega \varepsilon} \frac{\mathrm{d} B}{\mathrm{~d} z} \\
& E^{z}(\vec{Q}, \omega, z)=-\frac{\sin \theta_{i}}{\varepsilon} B
\end{aligned}
$$

Then we get the long wavelength limit $(\omega a / c \rightarrow 0)$ and following result (Bagchi and Kar 1978)

$$
\begin{aligned}
& \frac{E^{z}(Q \rightarrow 0, \omega, z)}{E_{0}}= \\
& \begin{cases}-\frac{\sin 2 \theta_{i}}{\left[\varepsilon(\omega)-\sin ^{2} \theta_{i}\right]^{1 / 2}+\varepsilon(\omega) \cos \theta_{i}}, & z \leqslant-a / 2 \\
-\frac{\sin 2 \theta_{i}}{z / a+a / 2[1+\varepsilon(\omega)] /[1-\varepsilon(\omega)]} \cdot & \frac{\varepsilon(\omega) /[1-\varepsilon(\omega)]}{\left[\varepsilon(\omega)-\sin ^{2} \theta_{i}\right]^{1 / 2}+\varepsilon(\omega) \cos \theta_{i}}, \\
-\frac{\sin 2 \theta_{i}}{\left[\varepsilon(\omega)-\sin ^{2} \theta_{i}\right]^{1 / 2}+\varepsilon(\omega) \cos \theta_{i}}, & z \geqslant a / 2\end{cases}
\end{aligned}
$$

We are mainly interested in the $z$-component of the electric field because for normal photoemission if the initial and final states are symmetric then $E^{z}$ is the component which will give a non-vanishing matrix element. 


\section{Results and discussions}

We have calculated the value of

$$
\tilde{A}_{\omega}(z)=\frac{E^{z}(Q \rightarrow 0, \omega, z)}{E_{0}}
$$

as a function of photon energy for various values of $z / a$ using the experimental values of $\varepsilon(\omega)$ (Weaver 1987-88). Figures $1,4,5,6$ and 7 represent the plots for $z / a=-0.5$, $0.0,0.5$. For each of the metals under study, we have plotted the $\left|\tilde{A}_{\omega}(z)\right|^{2}$ as this would be the quantity directly involved in the matrix element for the photocurrent calculations. We have taken the value of $\theta_{i}=45^{\circ}$. The thickness of the surface region $a$ is chosen arbitrarily. However for most metals $a=15 \AA$ with respect to the last plane of the atoms beyond which the electronic properties are independent of the presence of the surface (Appelbaum 1975). It has been found that for these metals no matter what values of $a$ are chosen (Thapa, unpublished results) the calculated electromagnetic field shows a minimum near the plasmon energy $\left(\hbar \omega_{p}\right)$ of the metals and a strong peak below $\hbar \omega_{p}$. For all these metals, the curve for $z / a=-0.5$ and 0.5 mimic the fields inside and outside the metal respectively that would be obtained if only Fresnel refraction were considered. The field for $z / a=0.0$ represents the behaviour in the surface region.

For $\mathrm{Al}$, the plot of $\tilde{A}_{\omega}(z)$ (and its square) against the photon energy $(h \omega)$ is shown in figure 1 for $z / a=-0.5,0.0$ and 0.5 . We find that the curves for $z / a=0.0$ and 0.5 show a minimum around $12 \mathrm{eV}$. This behaviour is in qualitative agreement with the experimental results of Levinson et al (1979). The calculated data of Levinson et al

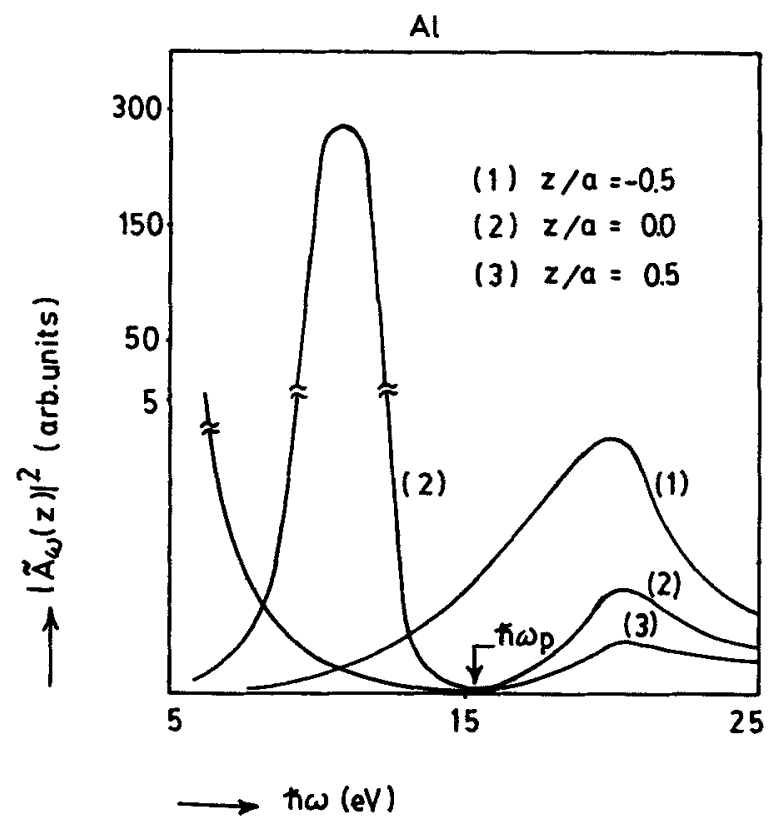

Figure 1. Variation of $\left|\tilde{A}_{\omega}(z)\right|^{2}$ plotted against the incident photon energy $(h \omega)$ for three planes parallel to the surface. (1) $z / a=-0 \cdot 5$, (2) $z / a=0 \cdot 0$ and (3) $z / a=0.5$. 
(1979) for normal photoemission cross-section from the surface state of $\mathrm{Al}$ is shown in figure 2. Thus the application of jellium model to $\mathrm{Al}$ is probably a better description. We also reproduce the experimental photoyield data of Petersen et al (1978) and that of Endriz (1973) and Barberan and Inglesfield (1981) in figure 3(b). We find that the data of Barberan and Inglesfield (1981) seems to be different than that of others.

We also considered metals for which jellium model would not be really applicable as these are not considered to be free electron metals. In figure 4, we show the case for $\mathrm{Ag}$ where again there is a minimum at the plasmon energy $(3.75 \mathrm{eV})$ and a strong peak just below that. Photoemission scattering cross-section calculation of $\mathrm{Ag}$ (Thapa, unpublished results) also showed similar behaviour giving evidence for the electromagnetic field results. This also seems to agree with the experimental photoemission data of Berguland and Spicer (1964) who have obtained a minimum in the quantum photoyield at around $3.75 \mathrm{eV}$ for Ag. However they attributed the transitions to take place from the $d$-band in $\mathrm{Ag}$ located approximately at $4 \mathrm{eV}$ below the Fermi level. The minimum in photoyield above $3.75 \mathrm{eV}$ was due to absorption of photons in exciting plasmons thus not producing photo-electrons.

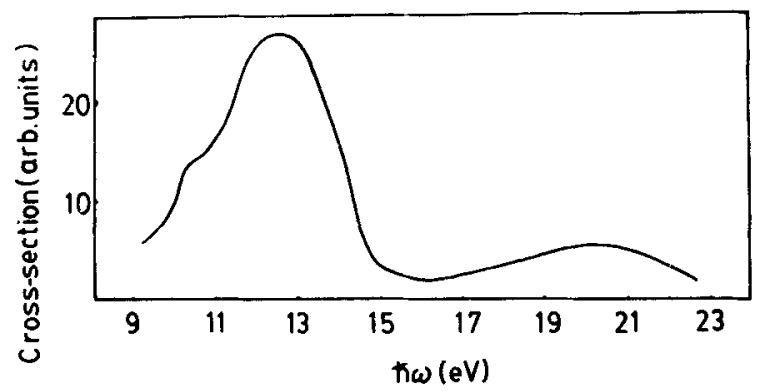

Figure 2. Calculated photoemission results as obtained by Levinson et al (1979) for $\mathrm{Al}$.

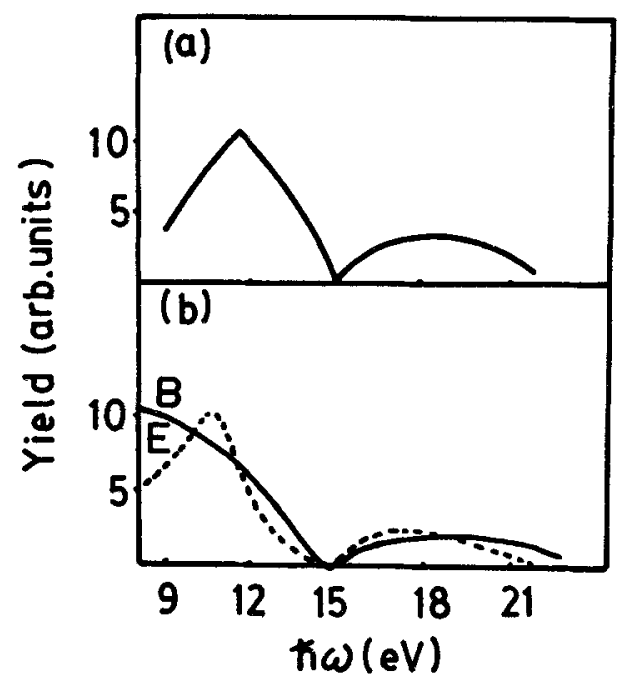

Figure 3. (a) The plot of experimental photoyield result for Al reproduced from Petersen et al (1978). (b) Photoemission yield from metal surface calculated via the matrix element by Barberan and Inglesfield (1981) (B) and Endriz (1973) (E). 
In figures 5, 6 and 7, we show the cases of $\mathrm{Cr}, \mathrm{Rh}$ and $\mathrm{Pd}$ respectively. Since the plasmons are not sharply defined for these metals, the structures are broadly the same with a shallow minimum for $z / a=+0.5$ and $z / a=0.0$ at the plasmon energy $\left(h \omega_{p}\right)$ and a peak below that. The curve for $z / a=-0.5$ behave in the same manner as the Fresnel field inside the metal. Very little theoretical and experimental data is available on the photoemission calculations of these metals. However, the photon energy dependence of electromagnetic field in the case of $\mathrm{Pd}$ had been reported perhaps for the first time by Thapa et al (1991).

Molybdenum shows a lot more structures as shown in figure 8 . The structures could be related to the dielectric function $\varepsilon(\omega)$, for example, a minimum in electromagnetic field occurs when the real part of $\varepsilon(\omega)$ changes sign but since $\varepsilon(\omega)$ themselves are experimentally determined quantities, we cannot make any firm

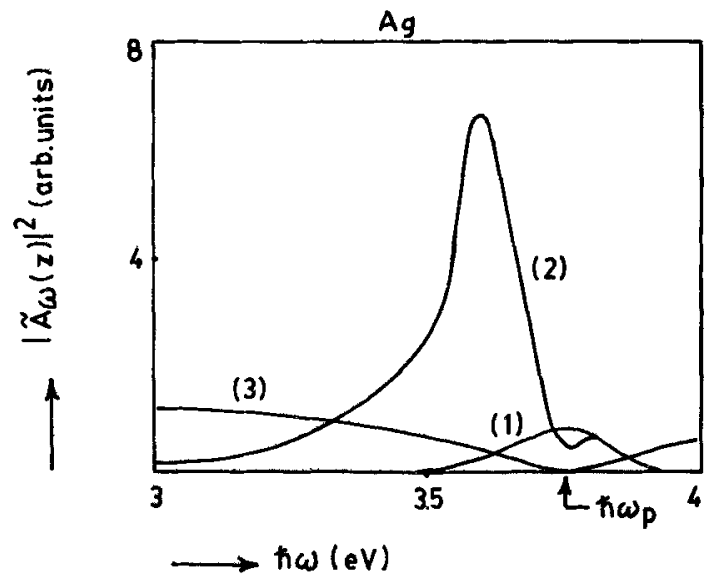

Figure 4. For caption, see p. 34.

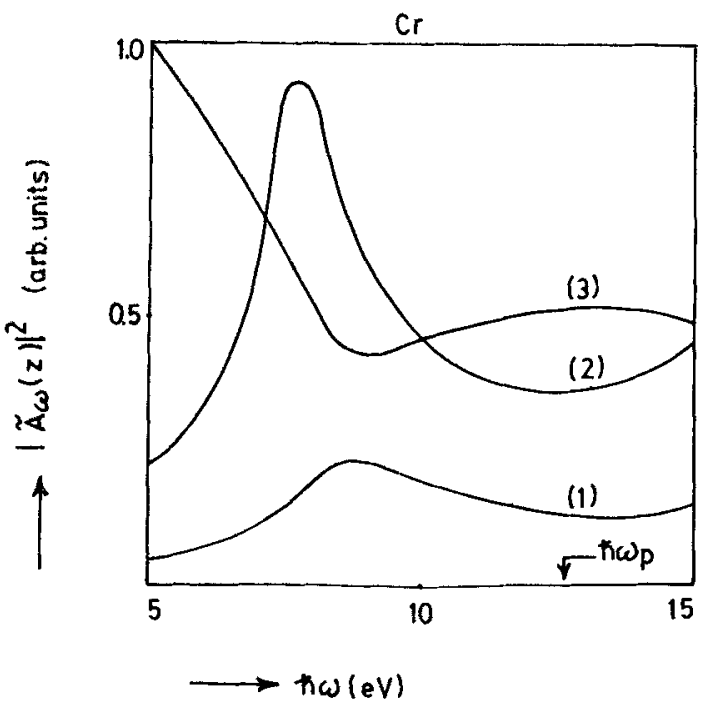

Figure 5. For caption, see p. 34. 

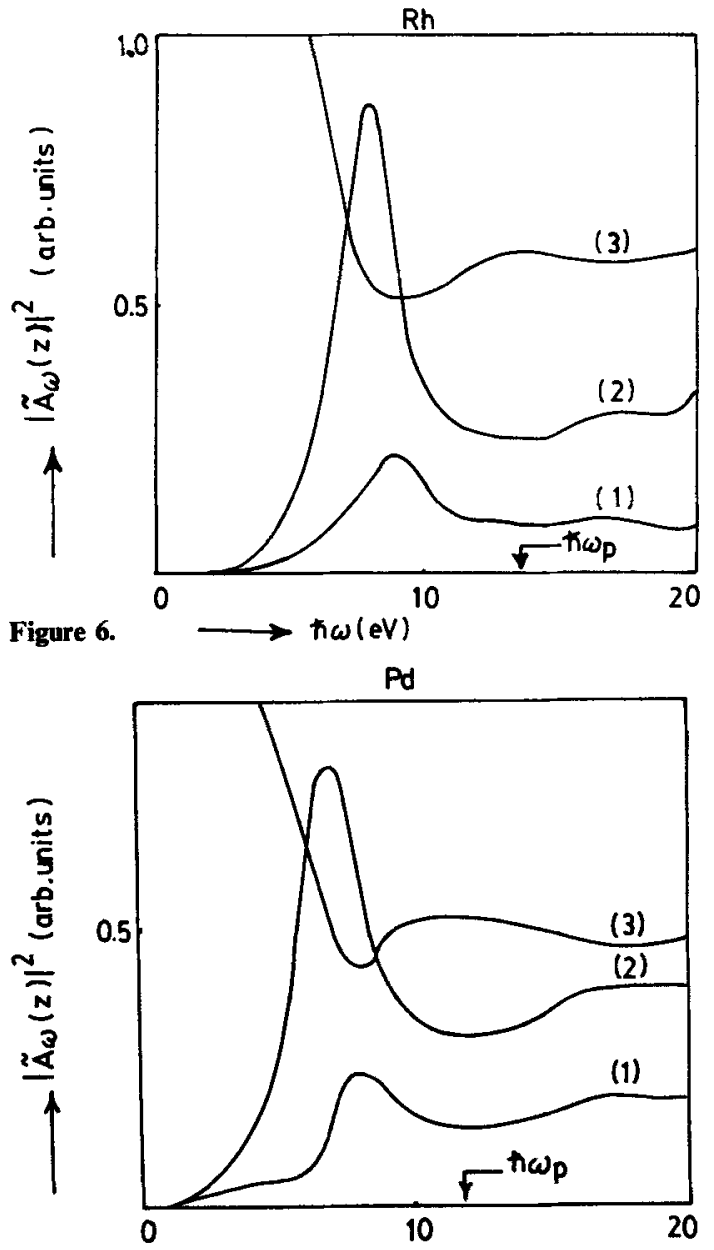

Figure 7. $\longrightarrow \hbar \omega(\mathrm{eV})$

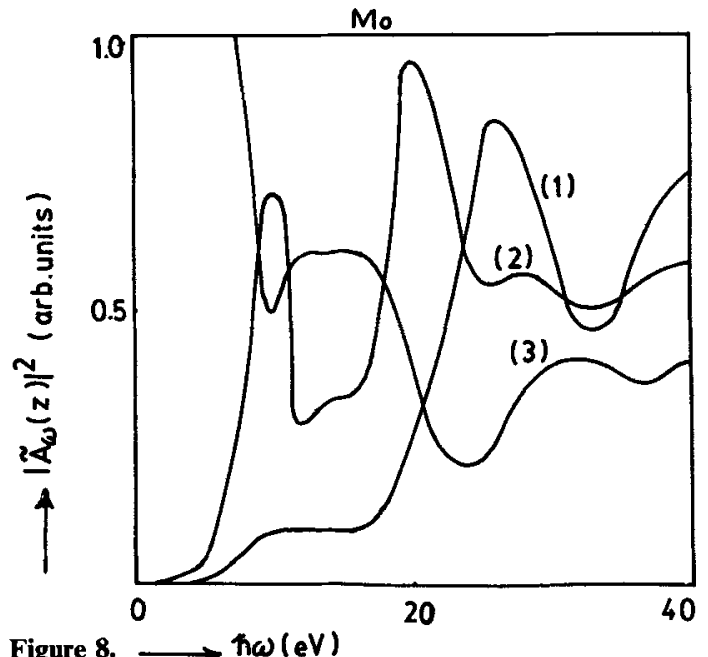

Figures 4-8. Variation of $\left|\tilde{A}_{\omega}(z)\right|^{2}$ plotted against the incident photon energy $(h \omega)$ for three planes parallel to the surface. (1) $z / a=-0.5$, (2) $z / a=0.0$ and (3) $z / a=0.5$. 

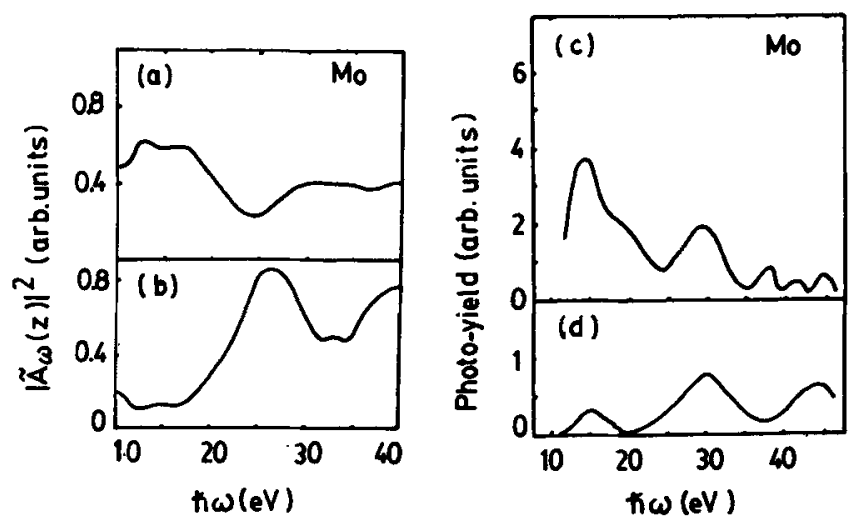

Figure 9. Photon energy dependence of electric field for (a) inside and (b) outside the surface of Mo reproduced from Weng et al (1978) and (ii) photoemission intensity from (a) high lying and (b) low lying surface resonance of Mo reproduced from Weng et al (1978).

statement about the origin of such structure. Weng et al (1978) have also done extensive studies of photoemission from Mo invoking refraction effects. Our results of Mo for regions within and outside the surface region seems to agree with the experimental data of Weng et al (1978). This is evident for the photon energy below and above the plasmon energy of $\mathrm{Mo}\left(h \omega_{p}=25 \mathrm{eV}\right)$.

\section{Conclusion}

The electromagnetic field used in this paper had been deduced using a very simple local dielectric model of the surface but yet the photocurrent calculations incorporating this field for bulk and surface state photoemission seem to agree well with the earlier published data (Thapa et al 1990; Das et al 1991). This model was also tested to see the effect of inclusion of the surface. For this the surface thickness was taken as $a=0$ and was applied to the case of Al. Neither the electromagnetic field did not show any peak for $\hbar \omega<\hbar \omega_{p}$ nor was there any suppression of the same at $\hbar \omega=\hbar \omega_{p}$. This implied that the surface plays a vital role during photoemission.

\section{Acknowledgement}

One of the authors (RKT) expresses his sincere gratitude to the Department of Physics, North Bengal University, Darjeeling for computational facilities, to Mr Rajan and Mr Raju of NIC Unit, Aizawl (Mizoram) for all their help while working with their computers, and to Dr Y N Tiwari, Department of Physics, Pachhunga University College, Aizawl for providing useful literature for this work from Patna University.

\section{References}


Berguland C N and Spicer W E 1964 Phys. Rev. A136 1044

Das P. Thapa R K and Kar N 1991 Modern Phys. Lett. B5 65

Endriz J G 1973 Phys. Rev. B7 3464

Feibelman P J 1975a Phys. Rev. B12 1319; 1975b Phys. Rev. Lett. 341092

Forstman F and Stenschke 1977 Phys. Rev. Lett. 381365

Kliewer K L 1976 Phys. Rev. B14 1412

Landau L D and Lifsitz E M 1984 The Electrodynamics of continuous media(New York: Pergamon Press) Sec-86, Problem No. 1

Levinson H J, Plummer E W and Feibelman P J 1979 Phys. Rev. Lett. 43952

Thapa R K, Kar N and Lal R A 1990 Indian J. Pure \& Appl. Phys. 294

Thapa R K, Das P and Kar N 1991 Phys. Teacher 3321

Weaver J H 1987-88 Handbook of chemistry and physics (Ohio: CRC Press) p. E-377

Weng S-L, Plummer E W and Gustafsson T 1978 Phys. Rev. B18 1718 